\title{
THE PERICELLULAR ENVIRONMENT REGULATES CYTOSKELETAL DEVELOPMENT AND THE DIFFERENTIATION OF MESENCHYMAL STEM CELLS AND DETERMINES THEIR RESPONSE TO HYDROSTATIC PRESSURE
}

\author{
Andrew J. Steward ${ }^{1,2,3}$, Diane R. Wagner ${ }^{3}$ and Daniel J. Kelly ${ }^{1,2, *}$ \\ ${ }^{1}$ Department of Mechanical and Manufacturing Engineering, School of Engineering, Trinity College Dublin, \\ Dublin, Ireland \\ ${ }^{2}$ Trinity Centre for Bioengineering, Trinity Biomedical Sciences Institute, Trinity College Dublin, Dublin, Ireland \\ ${ }^{3}$ Bioengineering Graduate Program, Department of Mechanical Engineering, University of Notre Dame, \\ Notre Dame, IN, USA
}

\begin{abstract}
The objective of this study was to examine the interplay between matrix stiffness and hydrostatic pressure (HP) in regulating chondrogenesis of mesenchymal stem cells (MSCs) and to further elucidate the mechanotransductive roles of integrins and the cytoskeleton. MSCs were seeded into $1 \%, 2 \%$ or $4 \%$ agarose hydrogels and exposed to cyclic hydrostatic pressure. In a permissive media, the stiffer hydrogels supported an osteogenic phenotype, with little evidence of chondrogenesis observed regardless of the matrix stiffness. In a chondrogenic media, the stiffer gels suppressed cartilage matrix production and gene expression, with the addition of RGDS (an integrin blocker) found to return matrix synthesis to similar levels as in the softer gels. Vinculin, actin and vimentin organisation all adapted within stiffer hydrogels, with the addition of RGDS again preventing these changes. While the stiffer gels inhibited chondrogenesis, they enhanced mechanotransduction of HP. RGDS suppressed the mechanotransduction of HP, suggesting a role for integrin binding as a regulator of both matrix stiffness and HP. Intermediate filaments also appear to play a role in the mechanotransduction of HP, as only vimentin organisation adapted in response to this mechanical stimulus. To conclude, the results of this study demonstrate that matrix density and/or stiffness modulates the development of the pericellular matrix and consequently integrin binding and cytoskeletal structure. The study further suggests that physiological cues such as HP enhance chondrogenesis of MSCs as the pericellular environment matures and the cytoskeleton adapts, and points to a novel role for vimentin in the transduction of HP.
\end{abstract}

Keywords: Biomechanics; cytoskeleton; differentiation; stem cells; chondrogenesis; hydrogel.

\footnotetext{
*Address for correspondence:

Daniel J Kelly, PhD

Department of Mechanical and Manufacturing Engineering, School of Engineering, Trinity College Dublin, Dublin 2, Ireland

Telephone Number: +353-1-8963947

FAX Number: +353-1-6795554

E-mail: kellyd9@tcd.ie
}

Multiple soluble and insoluble cues are known to regulate the differentiation of mesenchymal stem cells (MSCs), although we are only beginning to understand how these factors interact to regulate cell fate (Discher et al., 2009; Engler et al., 2006; Kelly and Jacobs, 2010; McBeath et al., 2004; Steward et al., 2011; Thorpe et al., 2012). The matrix or substrate stiffness has been shown to play a role in regulating the differentiation of MSCs down specific lineages in both 2D (Engler et al., 2006; Park et al., 2011) and 3D environments (Huebsch et al., 2010; Parekh et al., 2011; Pek et al., 2010). Softer substrates tend to guide MSCs down neurogenic, adipogenic and chondrogenic pathways, while stiffer substrates have been shown to support myogenesis and osteogenesis depending on the specific composition of the culture media (Engler et al., 2006; Huebsch et al., 2010; Liu et al., 2010; Park et al., 2011), although the underlying mechanisms by which stem cells sense and respond to substrate stiffness is not fully understood. Integrins form the linkage between a cell and its extracellular matrix (ECM) and have long been associated with mechanotransduction (Ingber, 2007), with matrix stiffness known to regulate integrin binding as well as the organisation of adhesion ligands (Huebsch et al., 2010). Inhibition of integrin binding disrupts modulusdriven differentiation in both 2D and 3D culture systems (Huebsch et al., 2010; Parekh et al., 2011; Park et al., 2011), confirming the role of integrin bonds in determining stem cell fate. It has also been shown that cytoskeletalintegrin linkage strength becomes stronger with increasing matrix elasticity (Choquet et al., 1997), implicating the cytoskeleton as a possible downstream target of matrix stiffness mechanotransduction. For example, myosin and actin-generated cytoskeletal tension are integral to mechanotransduction of substrate stiffness in 2D (Engler et al., 2006; McBeath et al., 2004), although the effects of actomyosin-generated cytoskeletal tension are less clear in 3D (Huebsch et al., 2010; Parekh et al., 2011).

In addition to cues generated in response to alterations in the stiffness and composition of the pericellular environment in vivo, stem cells will also be exposed to extrinsic mechanical loading which is known to influence their ultimate fate. The type (i.e. compression, fluid flow, tension, hydrostatic pressure), frequency, magnitude, and duration of loading all affect MSC lineage commitment (Haugh et al., 2011; Kelly and Jacobs, 2010; Meyer et al., 2011; Miyanishi et al., 2006b; Thorpe et al., 2008). Specifically, hydrostatic pressure (HP) is a key regulator 
of chondrogenesis (Elder and Athanasiou, 2009). HP has been shown to increase chondrogenic gene expression and matrix production in MSCs (Angele et al., 2003; Luo and Seedhom, 2007; Meyer et al., 2011; Miyanishi et al., 2006a; Miyanishi et al., 2006b; Ogawa et al., 2009; Steward et al., 2012; Wagner et al., 2008). HP also plays a role in maintaining the chondrogenic phenotype by suppressing the expression of type I collagen, alkaline phosphatase (ALP), matrix metalloproteinase 13 (MMP-13), type X collagen and Indian hedgehog (Ihh) (Steward et al., 2012; Vinardell et al., 2012; Wong et al., 2003). HP has been shown to disrupt actin stress fibre assembly in chondrocytes (Parkkinen et al., 1995), and inhibition of microtubules has been shown to suppress the beneficial effect of HP on chondrocyte matrix production in 2D culture (Jortikka et al., 2000). The exact mechanism through which HP is transduced is unknown. It has been proposed that increased HP would lead to an increase in entropy (under constant temperature and volume) by depolymerising cytoskeletal polymers into free monomers, therefore disrupting stress fibre assembly (Champeil et al., 1981; Crenshaw et al., 1996; Heremans, 1982; Mozhaev et al., 1996; Myers et al., 2007; Silva et al., 1996). Cell-matrix interactions have been shown to influence MSC response to HP during chondrogenesis in 3D culture, with a more robust response to loading observed in hydrogels that promoted stronger actin stress fibre formation (Steward et al., 2012). Similar to studies exploring stem cell response to matrix stiffness, these studies point to a role for integrins and cytoskeletal adaption in the mechanotransduction of HP.

The objective of this study was to examine the interplay between matrix stiffness and HP in regulating chondrogenesis of MSCs. Bone marrow-derived MSCs were encapsulated in hydrogels of differing stiffness and subjected to intermittent HP. Integrin binding was inhibited in some hydrogels in order to determine the role of integrin binding in the mechanotransduction of both matrix stiffness and HP. Actin microfilaments, microtubules, and intermediate filaments were also examined to determine their prospective roles in mechanotransduction. Our hypothesis was that softer hydrogels would support a more chondrogenic phenotype, but that changes in cell-matrix interactions and cytoskeletal development in the stiffer hydrogels would result in a more robust response to the application of HP.

\section{Materials and Methods}

\section{Cell isolation, expansion and encapsulation}

Bone marrow was harvested from the femoral diaphysis of 4-month-old pigs $(\sim 50 \mathrm{~kg})$ under sterile conditions. MSCs were isolated and expanded according to a modified method developed for human MSCs (Lennon and Caplan, 2006). Briefly, bone marrow was removed from the femur, washed and centrifuged twice, and sieved through a $40 \mu \mathrm{m}$ pore-size cell sieve (Falcon Starstedt, Wexford, Ireland). The remaining cell suspension was counted by trypan blue exclusion and seeded at a density of $10 \times 10^{6}$ cells per $175 \mathrm{~cm}^{2} \mathrm{~T}$-flask in a humidified atmosphere of $37^{\circ} \mathrm{C}$ and $5 \% \mathrm{CO}_{2}$. Non-adherent cells were removed after $3 \mathrm{~d}$ in culture to allow MSCs to attach to the flask. At each passage, cells were reseeded at a density of 875,000 cells per $175 \mathrm{~cm}^{2}$ T-flask. Cultures were expanded in highglucose Dulbecco's modified Eagle's Medium (hgDMEM GlutaMAX) supplemented with $10 \%$ foetal bovine serum (FBS), and penicillin (100 U/mL)-streptomycin $(100 \mu \mathrm{g} /$ $\mathrm{mL}$ ) (all GIBCO Biosciences, Dun Laoghaire, Ireland). After expansion (third passage), MSCs were encapsulated in agarose (Type VII, Sigma-Aldrich, Arklow, Ireland) at a density of $15 \times 10^{6}$ cells $/ \mathrm{mL}$. Briefly, MSCs were mixed with $5 \%$ agarose at $\sim 40{ }^{\circ} \mathrm{C}$ to yield final gel concentrations of $1 \%, 2 \%$ or $4 \%$ (with equilibrium moduli of $0.5,10$ and $25 \mathrm{MPa}$, respectively). The agarose-cell suspensions were cast in stainless steel moulds, and cored using biopsy punches to produce cylindrical scaffolds (Ø5 × $3 \mathrm{~mm}$ thickness). Constructs were maintained in $2.5 \mathrm{~mL} /$ construct of a chemically defined media (CDM) consisting of hgDMEM GlutaMAX supplemented with penicillin $(100 \mathrm{U} / \mathrm{mL})$-streptomycin $(100 \mu \mathrm{g} / \mathrm{mL})(\mathrm{GIBCO}$, Biosciences), $100 \mu \mathrm{g} / \mathrm{mL}$ sodium pyruvate, $40 \mu \mathrm{g} / \mathrm{mL}$ L-proline, $50 \mu \mathrm{g} / \mathrm{mL}$ L-ascorbic acid-2-phosphate, $1.5 \mathrm{mg} /$ $\mathrm{mL}$ bovine serum albumin (BSA), $1 \mathrm{x}$ insulin-transferrinselenium, $100 \mathrm{nM}$ dexamethasone (all Sigma-Aldrich) and either $16 \%$ FBS (permissive) or $10 \mathrm{ng} / \mathrm{mL}$ recombinant human transforming growth factor- $\beta 3$ (chondrogenic, TGF- $\beta 3$; ProSpec-Tany TechnoGene Ltd, Ness-Ziona, Israel). Some groups were also cultured with the addition of a $167 \mu \mathrm{M}$ RGDS peptide (RGDS+, Tocris Bioscience, Bristol, UK) in order to inhibit integrin binding. Cells that were to be cultured with the RGDS peptide were equilibrated in the chondrogenic media supplemented with RGDS for $2 \mathrm{~h}$ prior to encapsulation in agarose, and RGDS was added to the media during each further media change. Constructs were allowed to equilibrate overnight before the initiation of hydrostatic pressure.

\section{Application of hydrostatic pressure}

Constructs $(n=9)$ were sealed into sterile bags with $2 \mathrm{~mL}$ of medium per construct during the daily loading period. After loading, constructs were removed from the bags and returned to culture dishes containing $2.5 \mathrm{~mL}$ medium per construct to allow gases to equilibrate overnight. Cyclic HP was applied in a custom bioreactor filled with water within a $37^{\circ} \mathrm{C}$ incubator, as described previously (Meyer et al., 2011). The sealed bags exposed to HP were placed into the pressure vessel while the free swelling (FS) controls were placed into an open water bath next to the pressure vessel. The pressure vessel was connected to a hydraulic cylinder (PHD Inc., Fort Wayne, IN, USA) that was loaded using a computer controlled Instron 8874 materials testing machine. The pressure inside the vessel was measured using a pressure gauge (Omega Engineering Inc., Manchester, UK). The load applied to the hydraulic cylinder by the Instron was set such that the HP inside the vessel reached an amplitude of $10 \mathrm{MPa}$ at a frequency of $1 \mathrm{~Hz}, 4 \mathrm{~h} / \mathrm{d}, 5 \mathrm{~d} /$ week for 3 weeks. Half-medium exchanges were performed every 3-4 d and media samples were collected for biochemical analysis. 


\section{Biochemical Analysis}

Constructs $(n=4)$ were digested with papain $(125 \mu \mathrm{g} / \mathrm{mL})$ in $0.1 \mathrm{M}$ sodium acetate, $5 \mathrm{mM} \mathrm{L}$-cysteine- $\mathrm{HCl}$, and $0.05 \mathrm{M}$ EDTA (pH 6.0, all Sigma-Aldrich) at $60^{\circ} \mathrm{C}$ under constant rotation for $18 \mathrm{~h}$. Sulphated glycosaminoglycan (sGAG) content was quantified using the dimethylmethylene blue dye-binding assay (DMMB; Blyscan Biocolor Ltd., Carrickfergus, Northern Ireland) with a chondroitin sulphate standard. Collagen content was determined by measuring the hydroxyproline content. Samples were hydrolysed at $110{ }^{\circ} \mathrm{C}$ for $18 \mathrm{~h}$ in $38 \% \mathrm{HCl}$ and assayed using a chloromine-T assay with a hydroxyproline:collagen ratio of 1:7.69 (Ignat'eva et al., 2007; Kafienah and Sims, 2004). Media samples were also analysed using the DMMB and hydroxyproline assays, and subsequently added to that accumulated within constructs to yield the total sGAG and collagen produced. Total sGAG and hydroxyproline values from the HP groups were normalised to the FS groups when applicable. All assays were performed in triplicate.

\section{Confocal microscopy, histology and immunohistochemistry}

At day 21 , constructs $(n=2)$ were cut in half and fixed in $4 \%$ paraformaldehyde (Sigma-Aldrich) overnight at $4{ }^{\circ} \mathrm{C}$ and rinsed with PBS. In order to examine focal adhesion formation and cytoskeletal organisation, samples were permeabilised in a $1 \%$ Triton-X and $2 \%$ BSA solution for $45 \mathrm{~min}$ and washed in PBS. The samples were then incubated in a $1.5 \%$ BSA solution containing one of either $60 \mu \mathrm{g} / \mathrm{mL}$ monoclonal anti-vinculin FITC conjugate (Sigma-Aldrich), $5 \mathrm{U} / \mathrm{mL}$ rhodamine phalloidin (VWR International, Dublin, Ireland), $1 \mu \mathrm{g} / \mathrm{mL}$ anti-vimentin FITC (eBioscience, Inc., Hatfield, UK) or $1 \mu \mathrm{g} / \mathrm{mL}$ antialpha tubulin eFluor ${ }^{\circledR} 615$ (eBioscience, Inc.) for $1.5 \mathrm{~h}$, and then imaged using a Zeiss 510 Meta confocal microscope at $40 \mathrm{x}$ magnification.

The remaining halves were dehydrated and embedded in paraffin wax. Constructs were sectioned perpendicular to the disc face yielding $5 \mu \mathrm{m}$ thick sections. Sections were stained with either $1 \%$ alcian blue $8 \mathrm{GX}$ (SigmaAldrich) in $0.1 \mathrm{M} \mathrm{HCl}$ for $\mathrm{sGAG}$, or picro-sirius red to detect collagen. Collagen types I and II were further identified through immunohistochemistry. Sections were treated with peroxidase, followed by chondroitinase $\mathrm{ABC}$ (Sigma-Aldrich) in a humidified environment at $37^{\circ} \mathrm{C}$ for $1 \mathrm{~h}$ to permeabilise the extracellular matrix. Samples were then blocked with goat serum. Afterwards, the primary antibodies for collagen types I and II (mouse monoclonal, Abcam, Cambridge, UK) were applied for $1 \mathrm{~h}$. Next, the secondary antibody (anti-Mouse IgG biotin conjugate, Sigma-Aldrich) was added for $1 \mathrm{~h}$, followed by incubation with ABC reagent (Vectastain PK-4000, Vector Labs, Peterborough, UK) for $45 \mathrm{~min}$. Finally, the slides were developed with DAB peroxidase (Vector Labs) for $4 \mathrm{~min}$. Samples were washed with PBS between each step, and negative and positive controls of porcine ligament (positive for type I collagen, negative for type II collagen) and cartilage (positive for type II collagen, negative for type I collagen) were also assessed.
RNA isolation and quantitative real-time polymerase chain reaction

Quantitative real-time reverse transcription polymerase chain reaction (qRT-PCR) was used to determine relative gene expression changes in chondrogenic specific genes with respect to both application of loading and supplementation with RGDS peptide. Total RNA was extracted from agarose constructs $(n=3)$ directly after loading on day 14 of culture. Total RNA was extracted from each construct by homogenising each construct with an Ultra-turrax IKA T10 basic homogeniser (Fisher Scientific, Dublin, Ireland) in $1 \mathrm{~mL}$ of TRIZOL reagent (Invitrogen, Paisley, UK), followed by a chloroform (Sigma-Aldrich) extraction. The extracted solution was incubated with an equal volume of isopropanol and $5 \mu \mathrm{L}$ glycogen in a $-20^{\circ} \mathrm{C}$ freezer overnight. The solution was then centrifuged and the precipitate was washed once with $70 \%$ ethanol. The precipitate was resuspended in $200 \mu \mathrm{L}$ of $35 \%$ ethanol and the RNA was then extracted with a PureLink ${ }^{\mathrm{TM}}$ RNA Mini kit (Invitrogen) as per manufacturer's instructions. Total RNA yield and purity were analysed using an ND 1000 NanoDrop Spectrophotometer (Labtech International, Uckfield, UK) and adjusted to a standard concentration prior to cDNA synthesis. To quantify mRNA expression, 50 ng total RNA was reverse transcribed into cDNA using a high capacity reverse transcription cDNA kit (Applied Biosystems, Paisley, UK) as per manufacturer's instructions. TaqMan ${ }^{\circledR}$ gene expression assays (Applied Biosystems) which contain forward and reverse primers, and a FAM-labelled TaqMan probe for porcine Sox9 (Ss03392406 m1), aggrecan (Agc, Ss03374822 m1), collagen type II alpha 1 (Col2A1, Ss03373344_g1), and glyceraldehyde-3-phosphate dehydrogenase (GAPDH, Ss03373286) were used in this study. qRT-PCR was performed using an ABI 7500 sequence detection system (Applied Biosystems). $5 \mu \mathrm{L}$ cDNA preparation (diluted 1:5 with RNase free water), $1 \mu \mathrm{L}$ gene assay, $10 \mu \mathrm{L}$ TaqMan Universal PCR Master mix (Applied Biosystems) and $4 \mu \mathrm{L}$ RNase free water ( $20 \mu \mathrm{L}$ total volume) were added to each well. Samples were assayed in triplicate in one run ( 40 cycles). qRT-PCR data were analysed using the $\Delta \Delta \mathrm{C}_{\mathrm{T}}$ method as described previously (Livak and Schmittgen, 2001) with GAPDH as the endogenous control. Relative quantification values are presented as fold changes in gene expression relative to the control group.

\section{Statistical analysis}

Statistical analyses were performed using GraphPad Prism (version 5.00, GraphPad Software). Biochemical results, both numerical and graphical, are expressed in the form of mean \pm standard deviation. Differences between HP and FS samples or between samples cultured with or without RGDS were determined using a Student's $t$-test. A level of $p<0.05$ was considered significant. All sGAG, collagen, histological and confocal data are from samples collected on day 21. All gene expression data were collected on day 14 . 

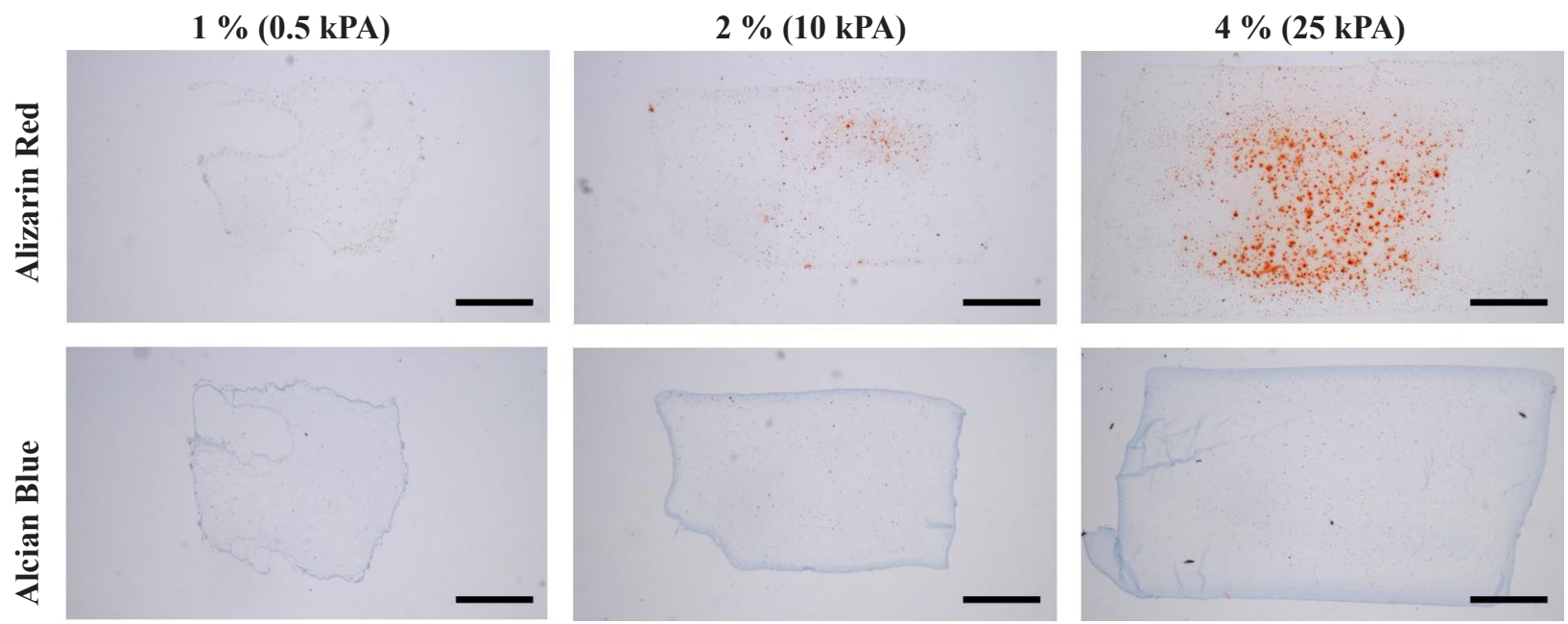

Fig. 1. Representative alcian blue and picro-sirius red images of MSCs cultured in a $16 \%$ FBS permissive media (scale bars $=1 \mathrm{~mm})$.

\section{Results}

\section{Influence of matrix stiffness on MSC differentiation in a permissive environment}

The Young's modulus of agarose hydrogels increases from $0.5 \mathrm{kPa}$ for $1 \%$ gels to $10 \mathrm{kPa}$ for $2 \%$ gels, to $25 \mathrm{kPa}$ for $4 \%$ gels. In order to assess how matrix stiffness affects differentiation of bone marrow-derived MSCs cultured in a permissive environment (Media $+16 \%$ FBS), histological sections were stained for calcific deposits and sGAG accumulation as markers of osteogenesis and chondrogenesis, respectively. With increasing matrix stiffness, more pronounced mineralisation was observed (Fig. 1). However, no evidence of chondrogenesis was observed in these specific culture conditions regardless of the local matrix stiffness (Fig. 1).

\section{Chondrogenesis of MSCs is regulated by matrix stiffness, integrin binding and cytoskeletal organisation}

Cell seeded constructs were maintained in a chondrogenic medium (Medium $+10 \mathrm{ng} / \mathrm{mL}$ TGF $\beta-3$ ) in order to assess the specific effects of matrix stiffness on the chondrogenesis of MSCs. Total sGAG and collagen production for each group was determined by summing the accumulation of specific matrix components within the hydrogels with that released into the medium. While sGAG accumulation within the constructs on day 21 was greater in the $4 \%$ agarose hydrogels, overall levels of both total sGAG (1 \%: $45.05 \pm 2.65 \mu \mathrm{g}, 2 \%$ : 33.60 $\pm 2.58 \mu \mathrm{g}, 4 \%: 29.39 \pm 1.32 \mu \mathrm{g})$ and collagen $(1 \%$ : $177.35 \pm 24.72 \mu \mathrm{g}, 2 \%$ : $84.04 \pm 7.00 \mu \mathrm{g}, 4 \%$ : 47.30 $\pm 3.00 \mu \mathrm{g}$ ) production decreased with increasing matrix stiffness (Fig. 2a,b). The pericellular environment was also found to depend on agarose hydrogel concentration, with a more well developed, intensely stained pericellular matrix (PCM, consisting of proteoglycans and collagens) observed in the stiffer hydrogels (Fig. 2e,f). To determine if interactions between MSCs and their local pericellular environment were regulating ECM synthesis, integrin binding was blocked with the addition of RGDS to the culture media. While RGDS had no significant effect on sGAG and collagen production in the softer $1 \%$ agarose hydrogels (Fig. 2c,d), inhibition of integrin binding led to a significant increase in ECM synthesis in the stiffer $4 \%$ agarose hydrogels, reaching levels comparable to those in $1 \%$ hydrogels (Fig. 2c,d).

We next sought to explore how matrix stiffness and associated changes in the pericellular environment influence both focal adhesion assembly and the cytoskeletal development of MSCs undergoing chondrogenesis. Staining for vinculin, a protein found in focal adhesions, was disperse in the softer $1 \%$ hydrogels, but had a more punctate appearance in the $4 \%$ hydrogels. This punctate structure was not evident with the addition of RGDS (Fig. 3 ). No visible changes in tubulin structure were observed with changes in matrix stiffness or the addition of RGDS, suggesting that the microtubule network is relatively insensitive to changes in the pericellular environment. The intensity of actin fluorescence increased with increasing matrix stiffness, with the addition of RGDS reducing the staining intensity. Staining for vimentin intermediate filaments became more punctate with increasing stiffness; and, as with vinculin staining, this punctate structure was less evident and staining more diffuse with the addition of RGDS (Fig. 3).

\section{Influence of hydrostatic pressure on chondrogenesis and the cytoskeletal organisation of MSCs}

While focal adhesion assembly, cytoskeletal organisation and cartilage-specific ECM synthesis are all regulated by the pericellular environment, in a developmental or regenerative context, MSCs will be additionally subjected to extrinsic mechanical forces in vivo such as hydrostatic pressure. How MSCs sense and respond to both intrinsic (i.e. those that are generated within the cell in response to the composition and stiffness of the PCM) and extrinsic (i.e. those generated from external mechanical loading) 
a

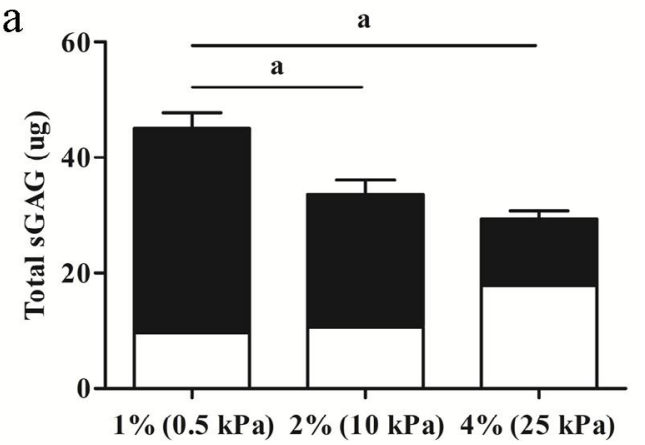

$\mathrm{C}$

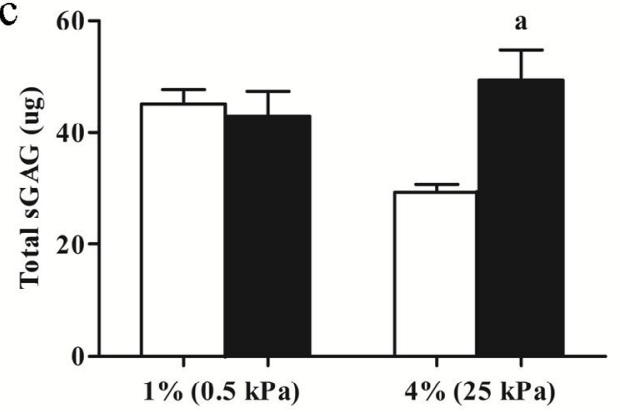

e
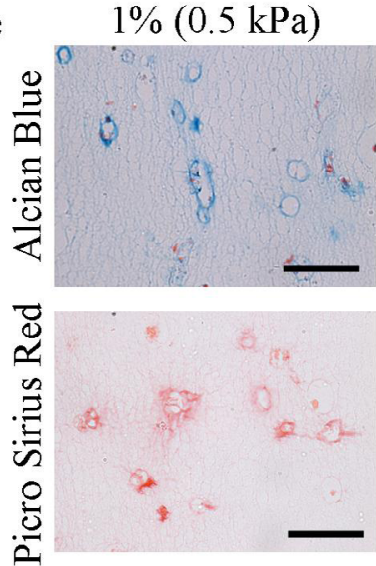

$\mathrm{b}$

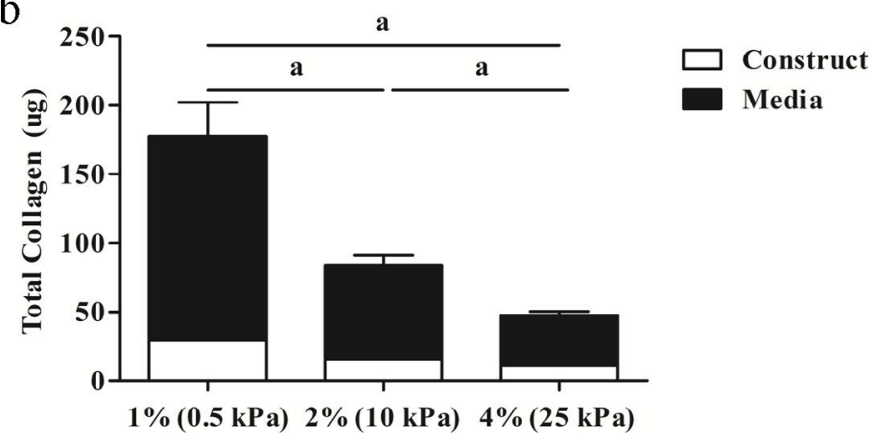

d

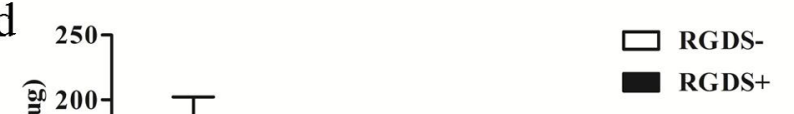

Fig. 2. (a) Total sGAG and (b) total collagen retained in the construct (white) and released to the media (black). (c) Total sGAG and (d) total collagen produced when cultured either with (RGDS+, black) or without (RGDS-, white) RGDS peptide. (e) Representative alcian blue and picro-sirius red images and (f) collagen type I and collagen type II histological and immunohistochemical images of $1 \%$ and $4 \%$ scaffolds (scale bars $=50 \mu \mathrm{m}$ ). a: $p<0.05$.

biophysical cues is poorly understood. The application of cyclic hydrostatic pressure (HP) was found to modulate ECM synthesis in a matrix stiffness dependent manner, with enhanced sGAG synthesis in response to HP (1\%: $0.99 \pm 0.14$ fold, $2 \%: 1.19 \pm 0.12$ fold, $4 \%: 1.42 \pm 0.23$ fold) only observed in the stiffer $4 \%$ hydrogels over $21 \mathrm{~d}$ of culture (Fig. 4a). Hydrostatic pressure had no effect on total collagen synthesis ( $1 \%: 0.88 \pm 0.14$ fold, $2 \%$ : 0.94 \pm 0.11 fold, $4 \%$ : $0.94 \pm 0.20$ fold) in any hydrogel (Fig. 4b). To further explore MSC response to HP in hydrogels of differing stiffness, the expression of a number of chondrogenic genes was analysed at day 14. Sox9, Agc and Col2A1 gene expression all increased significantly in the stiffer $4 \%$ hydrogels exposed to HP (Sox9: 1.41 \pm 0.20 fold, Agc: $1.32 \pm 0.13$ fold, Col2A1: $2.19 \pm 0.15$ fold), while HP had no positive effect on gene expression in the softer $1 \%$ hydrogels (Sox9: $0.90 \pm 0.17$ fold, Agc:
$0.43 \pm 0.46$ fold, Col2A1: $0.73 \pm 0.16$ fold) (Fig. $4 c-e$ ). Hydrostatic pressure had no visible effect on the intensity of vinculin, actin or tubulin staining or its localisation within the cell; however, the punctate structure of vimentin in the stiffer $4 \%$ hydrogels was no longer present after exposure to HP (Fig. 4f).

\section{Integrin binding is necessary for mechanotransduction of hydrostatic pressure} Given that integrin binding was required for MSCs to respond to changes in the stiffness and/or composition of their pericellular environment, we explored whether a similar pathway was involved in the mechanotransduction of HP. Addition of RGDS abrogated the beneficial response of HP on sGAG synthesis in the stiffer $4 \%$ hydrogels (-RGDS: $1.42 \pm 0.23$ fold, +RGDS: $0.90 \pm 0.11$ fold) over the $21 \mathrm{~d}$ culture period (Fig. 5a). Hydrostatic pressure had 

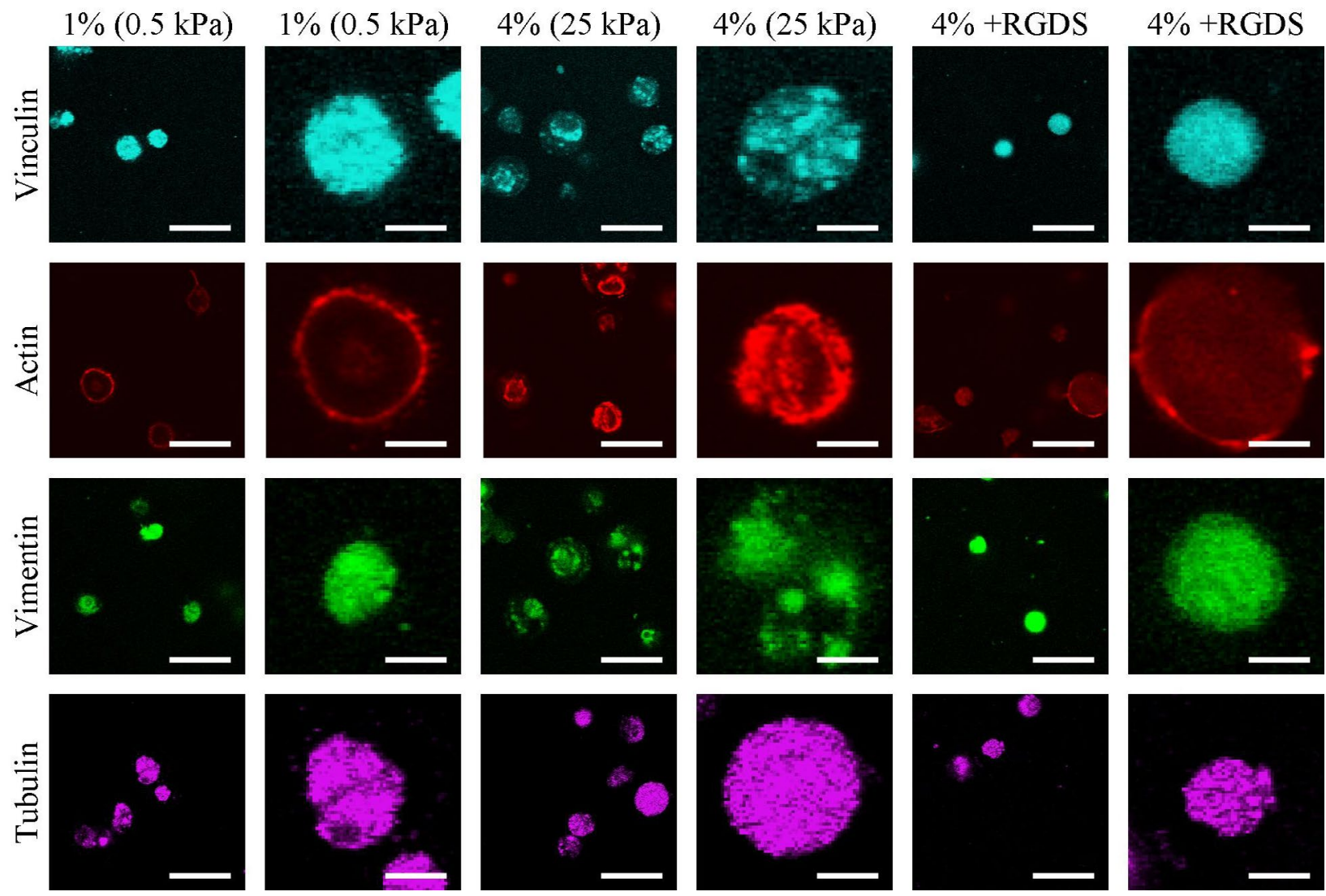

Fig. 3. Representative confocal images of vinculin, actin, vimentin and tubulin in $1 \%, 4 \%$ and $4 \%+$ RGDS constructs (scale bars $=50 \mu \mathrm{m}$ and $12.5 \mu \mathrm{m}$ ).

no effect on collagen production whether the media was supplemented with RGDS or not (-RGDS: $0.94 \pm 0.20$ fold, +RGDS: $1.21 \pm 0.33$ fold) (Fig. 5b). Furthermore, the increase in the expression of Sox 9, Agc and Col2A1 due to the application of hydrostatic pressure was also abolished in the presence of RGDS (Sox9: -RGDS: 1.41 \pm 0.20 fold, + RGDS: $1.26 \pm 0.27$ fold; Agc: - RGDS: 1.32 \pm 0.13 fold, + RGDS: $1.05 \pm 0.36$ fold; Col2A1: -RGDS: $2.19 \pm 0.15$ fold, +RGDS: $0.86 \pm 0.38$ fold) (Fig. $5 \mathrm{c}-\mathrm{e}$ ) In the presence of RGDS, no changes were observed in vinculin, actin, vimentin or tubulin due to the application of hydrostatic pressure (Fig. 5f).

\section{Discussion}

In agreement with previous studies seeding MSCs onto 2D substrates (Engler et al., 2006) or embedding them into 3D hydrogels (Huebsch et al., 2010; Parekh et al., 2011), we found that the stiffer $4 \%$ hydrogel supported a more osteogenic phenotype as evidenced by calcific deposits within constructs maintained in a permissive media that did not contain specific osteogenic supplements. Irrespective of hydrogel stiffness, this permissive medium did not support chondrogenesis of MSCs. As seen previously, when maintained in a medium supplemented with TGF- $\beta 3$, cartilage matrix production was inhibited in stiffer hydrogels (Bian et al., 2013; Erickson et al.,
2009). One potential explanation for this is that diffusivity of biomolecules (such as TGF- $\beta 3$ ) would be lower in the stiffer, denser $4 \%$ hydrogels; however, these hydrogels are still $96 \%$ fluid and are therefore not expected to significantly inhibit biomolecule diffusivity. While MSCs cannot directly adhere to agarose, and hence initially are unlikely to be able to sense their local stiffness, they rapidly synthesise fibronectin and other extracellular matrix components in hydrogel culture (Nicodemus et al., 2011; Parekh et al., 2011) to which they can adhere, which may provide them with a mechanism through which they can sense the stiffness of the surrounding hydrogel. In agreement with previous studies (Bian et al., 2013; Erickson et al., 2009), a denser, presumably stiffer, PCM also develops in the higher concentration hydrogels to which the MSCs can adhere and sense. In addition to a stiffer micro-environment for MSCs in the $4 \%$ hydrogels, it is also reasonable to assume that the more developed PCM in these constructs leads to an increase in the number of integrin binding sites per cell. It is therefore difficult to decouple whether the denser matrix exerts its effects via creating a stiffer pericellular environment, by increasing adhesion-ligand density (Connelly et al., 2008) or through some other feedback mechanism such as a change in the local charge density (or a combination of these factors). Previous studies have provided strong support for the hypothesis that MSCs interpret changes in the stiffness of their 3D pericellular environment as changes in adhesionligand presentation (Huebsch et al., 2010). 
a



$\mathrm{b}$

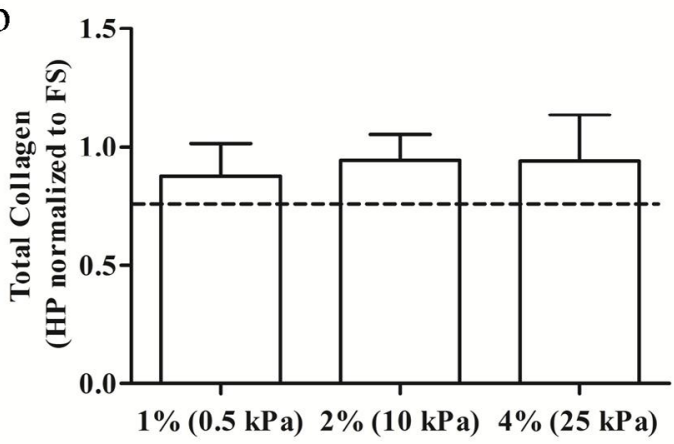

f $\quad 4 \% \mathrm{FS}$



$\mathrm{d}$

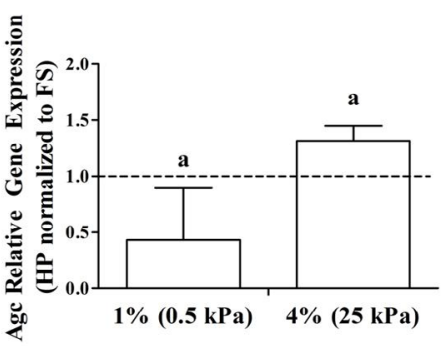

$\mathrm{e}$

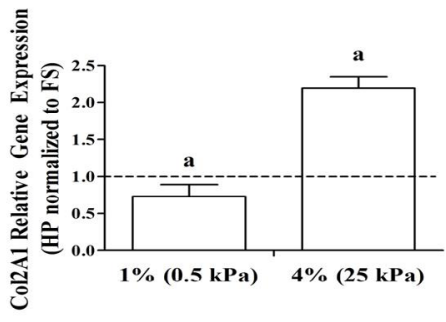

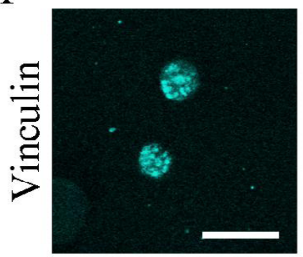
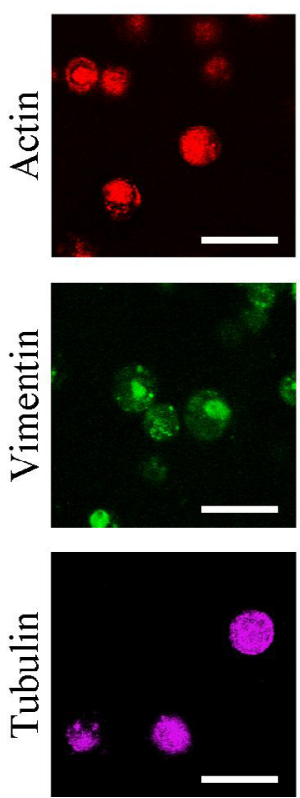
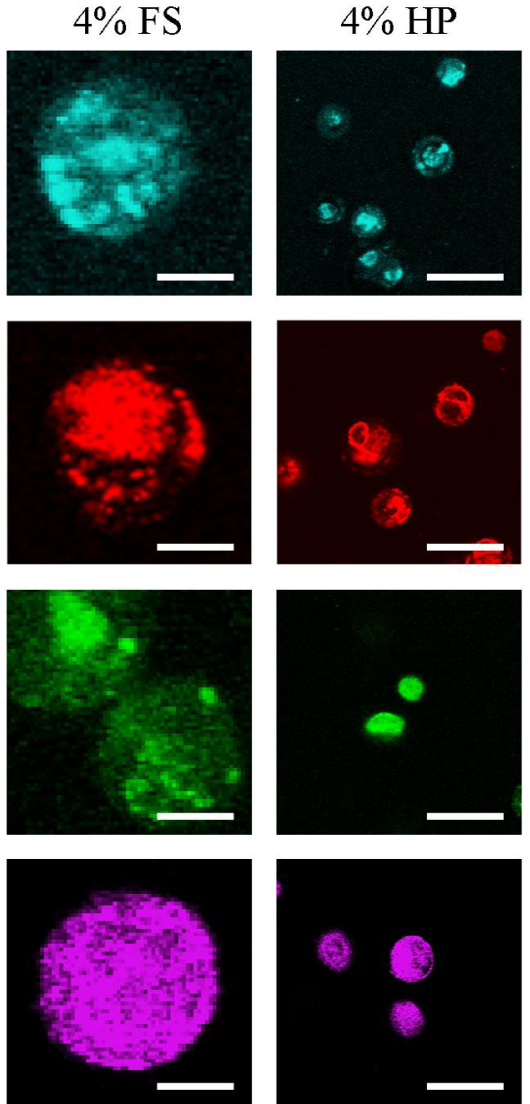
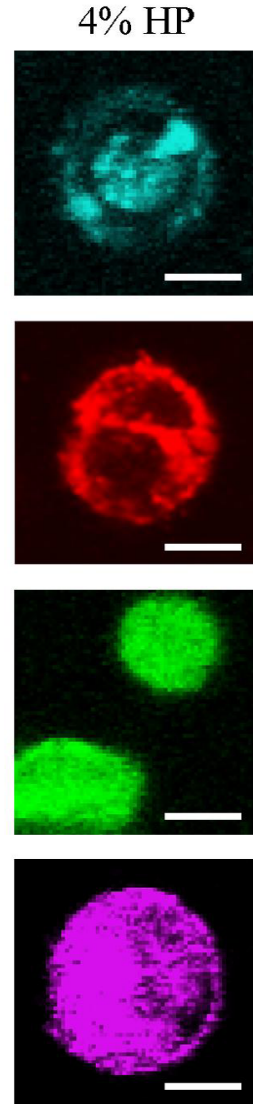

Fig. 4. (a) Total sGAG and (b) total collagen normalised to the FS condition. (c) Sox9, (d) Agc, and (e) Col2A1 relative gene expression normalised to the FS condition. (f) Representative confocal images of vinculin, actin, vimentin and tubulin in $4 \% \mathrm{FS}$ and HP groups (scale bars $=50 \mu \mathrm{m}$ and $12.5 \mu \mathrm{m}$ ). a: $p<0.05$.

The observed changes in MSC phenotype in the stiffer $4 \%$ hydrogels were accompanied by a more developed actin cytoskeleton. It is well established that development of actin stress fibres is correlated with an inhibition of chondrogenesis (Daniels and Solursh, 1991). Previous studies have also observed that conjugation of RGDadhesion ligands to agarose hydrogels inhibited sGAG synthesis by MSCs, and this inhibition could be blocked by the addition of a pharmacological actin inhibitor, further demonstrating that cellular adhesion and subsequent actin stress fibre formation inhibits chondrogenesis (Connelly et al., 2008). Based on these findings, we examined the roles of integrin binding and cytoskeletal organisation in the mechanotransduction of matrix stiffness. The addition of RGDS to the media, which blocks integrin binding to the PCM, impacted actin cytoskeleton development in the stiffer $4 \%$ gels and led to similar levels of ECM synthesis in these constructs relative to that observed in the softer hydrogels, further implicating integrin binding and actin as important elements in mechanotransduction. Vinculin, a component of focal adhesion complexes, exhibited a more punctate structure in the stiffer hydrogels, with the addition of RGDS leading to more diffuse staining, similar to that seen in the softer hydrogels. The more punctate organisation of vinculin in the stiffer hydrogels could indicate greater focal adhesion formation and FAK signalling, which has been shown to inhibit early chondrogenesis in MSCs (Pala et al., 2008). A punctate vimentin structure, perhaps indicative of filament formation, was observed in the stiffer $4 \%$ hydrogels, and again the addition of RGDS caused 
a

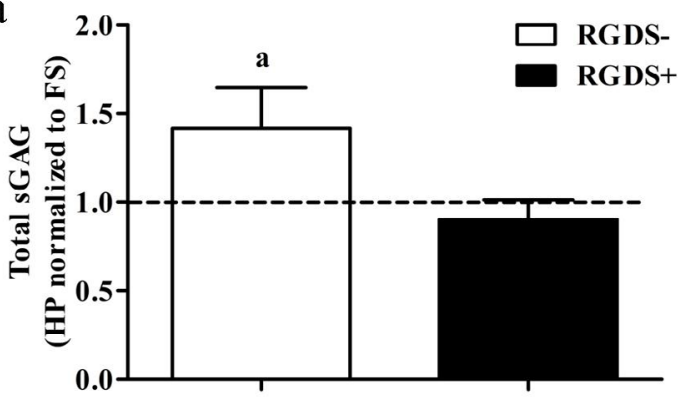

b



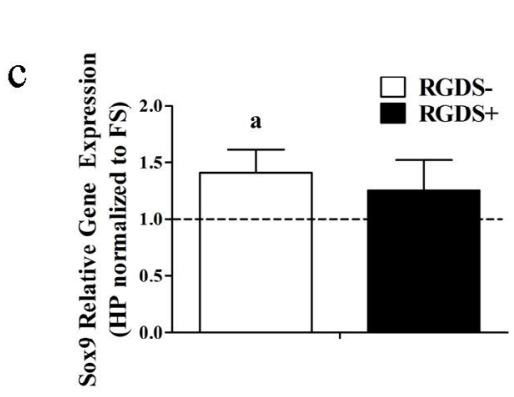

f $\quad 4 \% \mathrm{FS}+\mathrm{RGDS}$
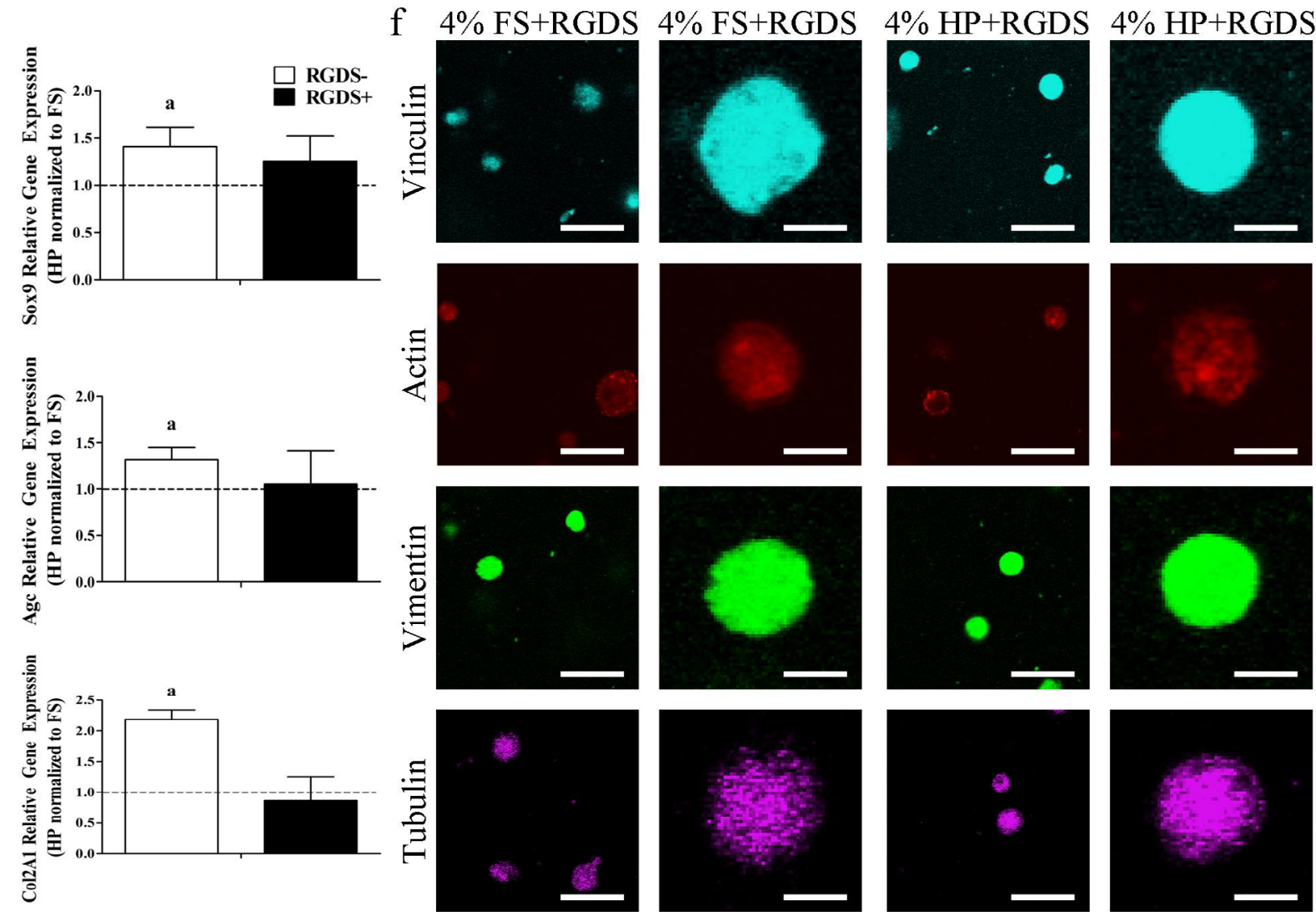

Fig. 5. (a) Total sGAG and (b) total collagen in $4 \%$ gels normalised to the FS condition. (c) Sox9, (d) Agc and (e) Col2A1 relative gene expression in $4 \%$ gels normalised to the FS condition. (f) Representative confocal images of vinculin, actin, vimentin and tubulin in $4 \% \mathrm{FS}$ and HP groups cultured with RGDS peptide (scale bars $=50 \mu \mathrm{m}$ and $12.5 \mu \mathrm{m})$. a: $p<0.05$.

the structure to resemble that in the softer $1 \%$ hydrogels. Vimentin is known to regulate chondrogenesis of MSCs, with siRNA-mediated knockdown of vimentin inhibiting cartilage-specific ECM production (Bobick et al., 2010). These intermediate filaments also contribute to the stiffness of chondrocytes (Haudenschild et al., 2011). Previous research has shown that vimentin can directly interact with actin, integrins $\alpha v \beta 3$ and $\alpha 2 \beta 1$, and their associated focal adhesions, which, together with the results of the current study, provides a mechanism by which vimentin may play a role in the transduction of mechanical cues (Esue et al., 2006; Gonzales et al., 2001; Kreis et al., 2005; Ruoslahti, 1996). The results of these and the present study demonstrate that although vimentin is critical for chondrogenesis, adaption of the intermediate filament network as the pericellular matrix becomes denser may play a role in the suppression of a chondrogenic phenotype in MSCs.

Prior studies have demonstrated that the application of HP can enhance chondrogenesis of MSCs (Elder and Athanasiou, 2009) and improve the mechanical functionality of tissue engineered cartilage (Liu et al., 2012; Meyer et al., 2011). Previously, we observed that the application of HP enhances chondrogenesis of MSCs in fibrin hydrogels where cells adopt a spread morphology with clear stress fibre formation, while MSCs embedded in agarose hydrogels remained rounded and did not respond to loading. These findings implicated cell shape and cytoskeletal dynamics in modulating the response of MSCs to HP (Steward et al., 2012). In this study, MSCs 
only responded to HP (as indicated by increases in the expression of certain chondrogenic genes and increased sGAG synthesis) in the stiffer $4 \%$ hydrogels, although MSCs retained a similar rounded morphology in both constructs, suggesting cell shape alone does not determine the response of MSCs to HP. Rather, it would appear that MSCs with clear focal adhesion assemblies, intense actin staining and a specific vimentin intermediate filament organisation respond anabolically to the application of HP. In such MSCs, HP would appear to disrupt the vimentin network, with more diffuse staining observed in MSCs exposed to HP, possibly implicating intermediate filaments in the mechanotransduction of HP. This diffuse staining may be indicative of depolymerisation of intermediate filaments due to the application of HP (Myers et al., 2007), which may occur due to an increase in entropy or alterations in phosphorylation pathways due to increased pressurisation (Crenshaw et al., 1996; Haskin and Cameron, 1993). Vimentin depolymerisation has been observed in certain cell types in response to HP (Crenshaw et al., 1996), with the same study reporting that microtubules appear more resistant to high levels of pressurisation. Induced swelling of cartilage explants was found to lead to vimentin disassembly, while swelling had no effect on actin organisation, further implicating an important mechanosensory role for vimentin in articular chondrocytes (Durrant et al., 1999). Together, these two studies, along with the current one, also suggest that different cytoskeletal elements may be more or less sensitive to different mechanical stimuli. The results of this study also provide further support for the concept that extrinsic mechanical cues can override the influence of the local substrate in determining MSC fate (Thorpe et al., 2012).

Given that chondrogenesis was suppressed in the stiffer $4 \%$ hydrogels via integrin-mediated binding to the pericellular matrix, and furthermore that the application of HP at least partially overcame this suppression of chondrogenesis, it seemed reasonable to assume that the response of MSCs to HP would be abrogated in the absence of integrin-mediated binding to the PCM. Indeed, HP had no effect on the expression of chondrogenic genes or cartilage specific matrix production in the presence of RGDS. One interpretation of this result is that MSCPCM integrin-mediated bonds are essential for the mechanotransduction of HP. Indeed, integrins have been implicated in the mechanotransduction of multiple extrinsic mechanical cues, including tension, compression, and fluid flow (Katsumi et al., 2004). However, the finding that HP had no influence on chondrogenesis with the addition of RGDS may not necessarily imply a direct role for integrins in the mechanosensing of HP, as blocking integrinmediated binding to the pericellular matrix also affected cytoskeletal components such as vimentin which may be the primary mechanosensors. The addition of RGDS in stiffer hydrogels led to the development of a cytoskeleton similar to that in the softer $1 \%$ hydrogels, where HP had no beneficial effect on chondrogenesis. For similar reasons, it is difficult to determine if changes in vimentin organisation are downstream or upstream of changes to integrin binding in the mechanotransduction pathway of
HP (i.e. RGDS alters both integrin binding and vimentin organisation, making it difficult to decouple their relative roles in the mechanotransduction of HP). Furthermore, the application of HP has no noticeable influence on the pattern of vinculin staining, although more quantitative analysis is required to definitively state that this mechanical cue is not influencing the composition or assembly of focal adhesions.

In conclusion, the pericellular matrix plays a crucial role in mechanotransduction of both HP and matrix stiffness through integrin binding and cytoskeletal organisation. MSCs embedded in the stiffer $4 \%$ hydrogels develop a more mature PCM, leading to changes in focal adhesion formation and cytoskeletal organisation and an inhibition of cartilage matrix synthesis and gene expression. The application of extrinsic mechanical cues, such as HP, can disrupt this process and override the influence of matrix stiffness on cytoskeletal development, promoting the maintenance of a chondrogenic phenotype as the pericellular environment matures and becomes stiffer. Integrin mediated binding to the PCM played a role in the mechanotransduction of both matrix stiffness and HP, although in the latter case downstream changes to the cytoskeleton following supplementation with RGDS make it impossible to state definitively that integrins are the primary mechanosensors of HP in MSCs. Finally, vimentin structure was also altered in the stiffer hydrogels when exposed to HP, suggesting a role for vimentin as a mechanotransductive element of HP.

\section{Acknowledgements}

This study was supported by a President of Ireland Young Researcher Award from the Science Foundation Ireland (08/Y15/B1336), funding from a European Research Council Starter Grant (No. 258463) and a Naughton Graduate Fellowship. We wish to confirm that there are no known conflicts of interest associated with this publication and there has been no significant financial support for this work that could have influenced its outcome.

\section{References}

Angele P, Yoo JU, Smith C, Mansour J, Jepsen KJ, Nerlich M, Johnstone B (2003) Cyclic hydrostatic pressure enhances the chondrogenic phenotype of human mesenchymal progenitor cells differentiated in vitro. J. Orthop Res 21: 451-457.

Bian L, Hou C, Tous E, Rai R, Mauck RL, Burdick JA (2013) The influence of hyaluronic acid hydrogel crosslinking density and macromolecular diffusivity on human MSC chondrogenesis and hypertrophy. Biomaterials 34: 413-421.

Bobick BE, Tuan RS, Chen FH (2010) The intermediate filament vimentin regulates chondrogenesis of adult human bone marrow-derived multipotent progenitor cells. J Cell Biochem 109: 265-276.

Champeil P, Bueschlen S, Guillain F (1981) Pressureinduced inactivation of sarcoplasmic reticulum adenosine 
triphosphatase high-speed centrifugation. Biochemistry 20: 1520-1524.

Choquet D, Felsenfeld DP, Sheetz MP (1997) Extracellular matrix rigidity causes strengthening of integrin-cytoskeleton linkages. Cell 88: 39-48.

Connelly JT, Garcia AJ, Levenston ME (2008) Interactions between integrin ligand density and cytoskeletal integrity regulate BMSC chondrogenesis. J Cell Physiol 217: 145-154.

Crenshaw HC, Allen JA, Skeen V, Harris A, Salmon ED (1996) Hydrostatic pressure has different effects on the assembly of tubulin, actin, myosin II, vinculin, talin, vimentin, and cytokeratin in mammalian tissue cells. Exp Cell Res 227: 285-297.

Daniels K, Solursh M (1991) Modulation of chondrogenesis by the cytoskeleton and extracellular matrix. J Cell Sci 100: 249-254.

Discher DE, Mooney DJ, Zandstra PW (2009) Growth factors, matrices, and forces combine and control stem cells. Science 324: 1673-1677.

Durrant LA, Archer CW, Benjamin M, Ralphs JR (1999) Organisation of the chondrocyte cytoskeleton and its response to changing mechanical conditions in organ culture. J Anat 194: 343-353.

Elder BD, Athanasiou KA (2009) Hydrostatic pressure in articular cartilage tissue engineering: from chondrocytes to tissue regeneration. Tissue Eng Part B Rev 15: 43-53.

Engler AJ, Sen S, Sweeney HL, Discher DE (2006) Matrix elasticity directs stem cell lineage specification. Cell 126: 677-689.

Erickson IE, Huang AH, Sengupta S, Kestle S, Burdick JA, Mauck RL (2009) Macromer density influences mesenchymal stem cell chondrogenesis and maturation in photocrosslinked hyaluronic acid hydrogels. Osteoarthritis Cartilage 17: 1639-1648.

Esue O, Carson AA, Tseng Y, Wirtz D (2006) A direct interaction between actin and vimentin filaments mediated by the tail domain of vimentin. J. Biol. Chem. 281: 30393 30399.

Gonzales M, Weksler B, Tsuruta D, Goldman RD, Yoon KJ, Hopkinson SB, Flitney FW, Jones JCR (2001) Structure and function of a vimentin-associated matrix adhesion in endothelial cells. Mol Biol Cell 12: 85-100.

Haskin C, Cameron I (1993) Physiological levels of hydrostatic pressure alter morphology and organization of cytoskeletal and adhesion proteins in MG-63 osteosarcoma cells. Biochem Cell Biol 71: 27-35.

Haudenschild DR, Chen J, Pang N, Steklov N, Grogan SP, Lotz MK, D'Lima DD (2011) Vimentin contributes to changes in chondrocyte stiffness in osteoarthritis. J Orthop Res 29: 20-25.

Haugh MG, Meyer EG, Thorpe SD, Vinardell T, Duffy GP, Kelly DJ (2011) Temporal and spatial changes in cartilage-matrix-specific gene expression in mesenchymal stem cells in response to dynamic compression. Tissue Eng Part A 17: 3085-3089.

Heremans K (1982) High pressure effects on proteins and other biomolecules. Annu Rev Biophys Bioeng 11: $1-21$.

Huebsch N, Arany PR, Mao AS, Shvartsman D, Ali OA, Bencherif SA, Rivera-Feliciano J, Mooney DJ (2010)
Harnessing traction-mediated manipulation of the cell/ matrix interface to control stem-cell fate. Nat Mater 9: 518-526.

Ignat'eva NY, Danilov NA, Averkiev SV, Obrezkova MV, Lunin VV, Sobol' EN (2007) Determination of hydroxyproline in tissues and the evaluation of the collagen content of the tissues. J Anal Chem 62: 51-57.

Ingber DE (2007) Integrins, tensegrity, and mechanotransduction. Gravitational Space Biol 10. 49-55.

Jortikka MO, Parkkinen JJ, Inkinen RI, Kärner J, Järveläinen HT, Nelimarkka LO, Tammi MI, Lammi MJ (2000) The role of microtubules in the regulation of proteoglycan synthesis in chondrocytes under hydrostatic pressure. Arch Biochem Biophys 374: 172-180.

Kafienah W, Sims TJ (2004) Biochemical methods for the analysis of tissue-engineered cartilage. Methods Mol Biol 238: 217-230.

Katsumi A, Orr AW, Tzima E, Schwartz MA (2004) Integrins in mechanotransduction. J Biol Chem 279: 12001-12004.

Kelly DJ, Jacobs CR (2010) The role of mechanical signals in regulating chondrogenesis and osteogenesis of mesenchymal stem cells. Birth Defect Res C 90: 75-85.

Kreis S, Schönfeld H-J, Melchior C, Steiner B, Kieffer $\mathrm{N}$ (2005) The intermediate filament protein vimentin binds specifically to a recombinant integrin $\alpha 2 / \beta 1$ cytoplasmic tail complex and co-localizes with native $\alpha 2 / \beta 1$ in endothelial cell focal adhesions. Exp Cell Res 305: 110121.

Lennon DP, Caplan AI (2006) Isolation of human marrow-derived mesenchymal stem cells. Exp Hematol 34: 1604-1605.

Liu SQ, Tian Q, Hedrick JL, Po Hui JH, Rachel Ee PL, Yang YY (2010) Biomimetic hydrogels for chondrogenic differentiation of human mesenchymal stem cells to neocartilage. Biomaterials 31: 7298-7307.

Liu Y, Buckley CT, Downey R, Mulhall KJ, Kelly DJ (2012) The role of environmental factors in regulating the development of cartilaginous grafts engineered using osteoarthritic human infrapatellar fat pad-derived stem cells. Tissue Eng Part A 18: 1531-1541.

Livak KJ, Schmittgen TD (2001) Analysis of relative gene expression data using real-time quantitative pcr and the 2- $\Delta \Delta C$ T method. Methods 25: 402-408.

Luo Z-J, Seedhom BB (2007) Light and low-frequency pulsatile hydrostatic pressure enhances extracellular matrix formation by bone marrow mesenchymal cells: An in-vitro study with special reference to cartilage repair. Proc Inst Mech Eng, Part H 221: 499-507.

McBeath R, Pirone DM, Nelson CM, Bhadriraju K, Chen CS (2004) Cell shape, cytoskeletal tension, and RhoA regulate stem cell lineage commitment. Dev Cell 6: 483-495.

Meyer EG, Buckley CT, Steward AJ, Kelly DJ (2011) The effect of cyclic hydrostatic pressure on the functional development of cartilaginous tissues engineered using bone marrow derived mesenchymal stem cells. J Mech Behav Biomed Mater 4: 1257-1265.

Miyanishi K, Trindade MCD, Lindsey DP, Beaupré GS, Carter DR, Goodman SB, Schurman DJ, Smith RL (2006a) Effects of hydrostatic pressure and transforming 
growth factor-beta 3 on adult human mesenchymal stem cell chondrogenesis in vitro. Tissue Eng. 12: 1419-1428.

Miyanishi K, Trindade MCD, Lindsey DP, Beaupré GS, Carter DR, Goodman SB, Schurman DJ, Smith RL (2006b) Dose- and time-dependent effects of cyclic hydrostatic pressure on transforming growth factor-beta3-induced chondrogenesis by adult human mesenchymal stem cells in vitro. Tissue Eng 12: 2253-2262.

Mozhaev VV, Heremans K, Frank J, Masson P, Balny C (1996) High pressure effects on protein structure and function. Proteins 24: 81-91.

Myers KA, Rattner JB, Shrive NG, Hart DA (2007) Hydrostatic pressure sensation in cells: integration into the tensegrity model. Biochem Cell Biol 85: 543-551.

Nicodemus GD, Skaalure SC, Bryant SJ (2011) Gel structure has an impact on pericellular and extracellular matrix deposition, which subsequently alters metabolic activities in chondrocyte-laden PEG hydrogels. Acta Biomater 7: 492-504.

Ogawa R, Mizuno S, Murphy GF, Orgill DP (2009) The effect of hydrostatic pressure on three-dimensional chondroinduction of human adipose-derived stem cells. Tissue Eng Part A 15: 2937-2945.

Pala D, Kapoor M, Woods A, Kennedy L, Liu S, Chen S, Bursell L, Lyons KM, Carter DE, Beier F, Leask A (2008) Focal adhesion kinase/Src suppresses early chondrogenesis: central role of CCN2. J Biol Chem 283: 9239-9247.

Parekh SH, Chatterjee K, Lin-Gibson S, Moore NM, Cicerone MT, Young MF, Simon Jr. CG (2011) Modulusdriven differentiation of marrow stromal cells in 3D scaffolds that is independent of myosin-based cytoskeletal tension. Biomaterials 32: 2256-2264.

Park JS, Chu JS, Tsou AD, Diop R, Tang Z, Wang A, Li $\mathrm{S}$ (2011) The effect of matrix stiffness on the differentiation of mesenchymal stem cells in response to TGF-[beta]. Biomaterials 32: 3921-3930.

Parkkinen JJ, Lammi MJ, Inkinen R, Jortikka M, Tammi M, Virtanen I, Helminen HJ (1995) Influence of short-term hydrostatic pressure on organization of stress fibers in cultured chondrocytes. J Orthop Res 13: 495-502.

Pek YS, Wan ACA, Ying JY (2010) The effect of matrix stiffness on mesenchymal stem cell differentiation in a $3 \mathrm{D}$ thixotropic gel. Biomaterials 31: 385-391.

Ruoslahti E (1996) RGD and other recognition sequences for integrins. Ann Rev Cell Develop Biol 12: 697-715.

Silva JL, Foguel D, Da Poian AT, Prevelige PE (1996) The use of hydrostatic pressure as a tool to study viruses and other macromolecular assemblages. Curr Opin Struct Biol 6: 166-175.

Steward AJ, Liu Y, Wagner DR (2011) Engineering cell attachments to scaffolds in cartilage tissue engineering. JOM 63: 74-82.

Steward AJ, Thorpe SD, Vinardell T, Buckley CT, Wagner DR, Kelly DJ (2012) Cell-matrix interactions regulate mesenchymal stem cell response to hydrostatic pressure. Acta Biomater 8: 2153-2159.

Thorpe SD, Buckley CT, Vinardell T, O'Brien FJ, Campbell VA, Kelly DJ (2008) Dynamic compression can inhibit chondrogenesis of mesenchymal stem cells. Biochem Biophys Res Commun 377: 458-462.

Thorpe SD, Buckley CT, Steward AJ, Kelly DJ (2012) The external mechanical environment can override the influence of local substrate in determining stem cell fate. J Biomech 45:2483-2492.

Vinardell T, Rolfe RA, Buckley CT, Meyer EG, Ahearne M, Murphy P, Kelly DJ (2012) Hydrostatic pressure acts to stabilise a chondrogenic phenotype in porcine joint tissue derived stem cells. Eur Cell Mater 23: 121-134.

Wagner DR, Lindsey DP, Li KW, Tummala P, Chandran SE, Smith RL, Longaker MT, Carter DR, Beaupre GS (2008) Hydrostatic pressure enhances chondrogenic differentiation of human bone marrow stromal cells in osteochondrogenic medium. Ann Biomed Eng 36: 813820 .

Wong M, Siegrist M, Goodwin K (2003) Cyclic tensile strain and cyclic hydrostatic pressure differentially regulate expression of hypertrophic markers in primary chondrocytes. Bone 33: 685-693.

\section{Discussion with Reviewers}

Reviewer I: A discussion on the potential effects of HP on the agarose hydrogel properties would be useful.

Authors: We presume the reviewer is referring to the mechanical properties of the hydrogels. Based on research previously performed in our lab, hydrostatic pressure has been found to significantly increase the dynamic modulus of agarose hydrogels in a time- and donor-dependent manner (Meyer et al., 2011). For example, we have previously shown that the dynamic modulus of MSC-seeded agarose hydrogels is unaffected by the application of HP at day 21 , but on day 42 of culture, loaded hydrogels were found to have a significantly higher dynamic modulus than free swelling (FS) controls. In agreement with this data, HP did not significantly affect the mechanical properties of cell-seeded agarose hydrogels on day 21 relative to FS controls in the current study (data not shown).

Reviewer II: The work of Connelly et al, who showed that appending RGD ligands to agarose resulted in a fundamental shift in differentiation response. It seems that the response here, being mediated by pericellular accumulation, would be informed by these previous findings. Also, aside from stiffness-mediated effects, could not feed-back inhibition (via charge density) play a role? Please comment.

Authors: It is true that the findings of this study can be related to the study performed by Connelly et al. (2008). They observed that conjugation of the RGD peptide to agarose hydrogels decreased chondrogenesis in an RGD density dependant manner and that disruption of the actin cytoskeleton suppressed this effect. As already discussed, the suppression of chondrogenesis in the stiffer/denser $4 \%$ hydrogels observed in this study could be due to a greater accumulation of PCM molecules, and this higher density of binding sites could lead to a suppression of 
chondrogenesis in a similar manner to that observed by Connelly et al. (2008). A feedback inhibition (via charge density) has been included as another potential mechanism for this result in the discussion section.

Reviewer II: The authors note: "While MSCs cannot directly adhere to agarose, and hence initially are unlikely to be able to sense their local stiffness, they rapidly synthesise fibronectin and other extracellular matrix components in hydrogel culture (Nicodemus et al., 2011; Parekh et al., 2011), to which they can adhere", and suggest that this provides a mechanism by which the cells can sense the hydrogel stiffness. I am not sure that I understand this proposed mechanism. Do the authors believe that the formed material forms an interpenetrating network with the agarose, and that cell contraction then allows them to 'pull' on the agarose? Or, instead, does the denser hydrogel 'compact' the material in a smaller space, increasing the density of ligands for integrin and placing a compressive 'pre-stress' on the cell? Isn't this what happens in traditional pellet culture, perhaps without the compressive pre-stress? Is the response to HP in pellet culture different from that at high agarose densities? The authors should perform studies to elucidate these potentially competing aspects.

Authors: Clearly the PCM is more developed in the stiffer/ denser $4 \%$ hydrogels, and hence one could reasonably expect the density of ligands to be higher in these constructs. This denser PCM is presumably also stiffer and the cells can 'pull' on it. In addition, it is also possible that the synthesised matrix forms an 'interpenetrating network with the agarose', allowing the MSCs to sense the hydrogel stiffness, although it is difficult to test this hypothesis directly. Regardless of the underlying mechanisms, it would seem reasonable to conclude that a stiffer cellular micro-environment exists in the $4 \%$ agarose hydrogels which the cells can sense. Of course, as we have already discussed, as with any hydrogel system (whether ligands are conjugated or not) it is difficult to decouple the effects of matrix stiffness and ligand density in long-term culture as the hydrogel stiffness/density will impact PCM formation and hence alter ligand density. To summarise, what would seem reasonable to conclude is that the MSC micro-environment in the $1 \%$ gels is less stiff and over the $21 \mathrm{~d}$ culture period presents a lower density of ligands for integrin attachment, while the $4 \%$ gels provide a stiffer microenvironment and over time a higher density of ligands as the PCM develops faster. Regarding the comment on pellet culture, we and others have shown that the application of HP to MSC pellets enhances chondrogenesis and matrix synthesis over a 2-3 week culture period. This is a similar response to that observed in the stiffer/denser agarose hydrogels. 